syndrome (TS) patients (mean age, 41 years), with 10 normal volunteers as controls, in a study at North Shore University Hospital, Manhasset, New York. Quantitative fluorodeoxyglucose (FDG) and PET were used to calculate global and regional rates of glucose metabolism. While global glucose metabolic rates were normal in TS patients, SSM analysis identified bilateral metabolic increases in lateral premotor and supplementary motor cortex and midbrain, and decreases in caudate and thalamic metabolism. TS patients have a nonspecific pattern of increased motor cortical activity common to other hyperkinetic disorders, and a specific brain network involving reduced activity of limbic basal ganglia-thalamocortical projections. (Eidelberg D, Moeller JR, Antonini A et al. The metabolic anatomy of Tourette's syndrome. Neurology April 1997;48:927-934). (Reprints: Dr D Eidelberg, Movement Disorders Center, North Shore University Hospital, 444 Community Drive, Manhasset, New York, 11030).

COMMENT. Two independent brain networks are identified in patients with Tourette syndrome: 1) increased metabolic activity of motor cortical regions involved in execution of movement; and 2) decreased metabolic activity in basal ganglia-thalamic areas governing TS global scale severity.

\title{
GENETICS OF TOURETTE SYNDROME
}

Age at onset, age at diagnosis, and phenotypic expressions of TS were compared in the offspring of affected males and females among 437 first degree relatives of 57 probands studied at Academic Department of Psychiatry, University College London Medical School, London, UK. Maternally transmitted cases had an earlier age at onset, suggesting a genomic imprinting effect on the expression of TS. (Eapen V, O'Neill J, Gurling HMD, Robertson MM. Sex of parent transmission effect in Tourette's syndrome: Evidence for earlier age at onset in maternally transmitted cases suggests a genomic imprinting effect. Neurology April 1997;48:934-937). (Reprints: Dr V Eapen, Faculty of Medicine \& Health Sciences, United Arab Emirates University, PO Box 17666, Al Ain, United Arab Emirates).

COMMENT. Family history data on Tourette syndrome cases must be evaluated for maternal or paternal transmission. Mothers affected with TS are likely to have affected offspring with an earlier age at onset. Environmental factors, such as exposure to stress or cocaine, may be alternative explanations for this finding.

Developmental Basal Ganglia Syndrome. Failure to distinguish TS from other causes of DBGS may explain confusion in localizing the genetic defect in TS, determining prevalence, and assessing therapy, according to a report from the University of Rochester, NY. (Palumbo D, Maughan A, Kurlan R. Hypothesis III. Tourette syndrome is only one of several causes of developmental basal ganglia syndrome. Arch Neurol April 1997;54:475-483). Causes of the DBGS include primary (hereditary) tic disorders, obsessivecompulsive disorder, ADHD, Huntington disease, and secondary (symptomatic) autism, mental retardation, intrauterine drug exposure, perinatal asphyxia, encephalitis, head trauma, etc.

\section{STIMULANT THERAPY OF ADHD AND TOURETTE SYNDROME}

The effects of methylphenidate (MPH) and dextroamphetamine (DEX) on tic severity in 20 boys with ADHD and comorbid Tourette syndrome were investigated in a 9-week, placebo-controlled, double-blind crossover study at 